\title{
Ganho de peso e comportamento de oviposição de Podisus nigrispinus utilizando lagartas de Spodoptera frugiperda e larvas de Tenebrio molitor como presas
}

\author{
Body increment and oviposition behavior of Podisus nigrispinus by utilizing \\ Spodoptera frugiperda and Tenebrio molitor larvae preys
}

\author{
Harley Nonato de Oliveira ${ }^{1}$ Marcelo Curitiba Espindula ${ }^{2}$ \\ Dirceu Pratissoli ${ }^{3}$ Eder Pin Pedruzzi ${ }^{2}$
}

\begin{abstract}
RESUMO
Esse trabalho avaliou o efeito de diferentes presas e da combinação destas sobre percevejo Podisus nigrispinus. $O$ delineamento experimental foi inteiramente casualizado com três tratamentos e 60 repetições. No tratamento um (T1), os percevejos receberam como alimento, lagartas de Spodoptera frugiperda, de 4o estádio, durante todo o seu ciclo de vida, no tratamento dois (T2), larvas de Tenebrio molitor, também durante o todo ciclo, enquanto que, no tratamento três (T3), foram oferecidas lagartas de $\boldsymbol{S}$. frugiperda do $2^{\circ}$ ao $4^{\circ}$ estádios, e larvas de $\mathbf{T}$. molitor do $4^{\circ}$ estádio até o final do ciclo de vida. O ganho de peso em todas as fases ninfais e em adultos de até terceiro dia mostrou valores semelhantes de incremento, para todas as dietas utilizadas. No entanto, para os percevejos alimentados, com $\boldsymbol{S}$. frugiperda (T1), observaram-se uma maior produção de ovos num menor período, com $80 \%$ dos ovos até $31^{\circ}$ dia, enquanto que, no tratamento com larvas de T. molitor (T2), os mesmos $80 \%$ foram conseguidos somente no $45^{\circ}$ dia, e no tratamento com a combinação de presas (T3), no 48ํ dia.
\end{abstract}

- NOTA -
Palavras-chaves: controle biológico, predadores, inimigo natural.

\section{ABSTRACT}

The objective of this research was to evaluate the effect of different kind of preys and their combination when offered to Podisus nigrispinus. The bioassay was composed of three treatments with sixty replicates each, in a randomized complete block design. Treatment one (T1) consisted of $\boldsymbol{P}$. nigrispinus feeding on $4^{\text {th }}$ instar of Spodoptera frugiperda larvae; treatment two (T2) consisted of Tenebrio molitor larvae only, and treatment three (T3) consisted of $2^{\text {nd }}$ to $4^{\text {th }}$ instar of $S$. frugiperda larvae and $\mathbf{T}$. molitor larvae until the end of the $\mathbf{P}$. nigrispinus life cycle. The body weight in all nymphal instars and in 3 day old adults were the same with all preys used. However, predators fed with $S$. frugiperda (T1) showed a greater number of eggs in a smaller period, with $80 \%$ of the eggs laid up to the $31^{\text {st }}$ day, while $\boldsymbol{P}$. nigrispinus fed on T. molitor (T2) the same rate was achieved up to the $45^{\text {th }}$ day, and when a combination of preys were used (T3), the rate was achieved up to the $48^{\text {th }}$ day.

Key words: biological control, predators, natural enemy.

O percevejo predador Podisus nigrispinus (Dallas) (Heteroptera: Pentatomidae) é uma espécie que ocorre desde a Argentina até a Costa Rica (THOMAS, 1992), sendo citado no Brasil como inimigo natural de pragas agrícolas e florestais (GRAVENA \& LARA, 1982; ZANUNCIO et al., 1993). Apesar de já estar sendo produzido e liberado em plantações de eucalipto no Brasil (ZANUNCIO et al., 2002), aspectos biológicos de $\boldsymbol{P}$. nigrispinus têm sido estudados visando otimizar a criação massal desse inseto, pois o mesmo apresenta potencial para o

\footnotetext{
${ }^{1}$ Engenheiro Agrônomo, Doutor., Bolsista PROFIX do Conselho Nacional de desenvolvimento Científico e Tecnológico (CNPq) Brasil, Centro de Ciências Agrárias (CCA), Universidade Federal do Espírito Santo (UFES), Laboratório de Entomologia, CP 16, 29500-000, Alegre, ES. E-mail: hnoliveira@insecta.ufv.br.

${ }^{2}$ Estudante de Agronomia, Bolsista de Iniciação Científica do (CNPq), CCA, UFES.

${ }^{3}$ Engenheiro Agrônomo, Doutor, Professor Adjunto, Departamento de Fitotecnia, CCA, UFES.
} 
controle biológico muito além do utilizado atualmente (TORRES et al., 1996).

O alimento é um componente importante do meio, pois influi diretamente na distribuição e abundância dos insetos, e afeta processos biológicos como fecundidade, longevidade, velocidade de desenvolvimento e comportamento (ZANUNCIO et al., 1991). Assim princípios nutricionais podem ser aplicados para aumentar o vigor, a performance e a efetividade de inimigos naturais contra as pragas (HOUSE, 1977).

Este trabalho avaliou o ganho de peso e comportamento de oviposição de Podisus nigrispinus, quando alimentados com lagartas de Spodoptera frugiperda (Lepidoptera: Noctuidae), larvas de Tenebrio molitor (Coleoptera: Tenebrionidae) e com a combinação destas presas.

O experimento foi conduzido no laboratório de Entomologia do Centro de Ciências Agrárias da Universidade Federal do Espírito Santo a $25 \pm 2^{\circ} \mathrm{C}$, umidade relativa de $70 \pm 10 \%$, fotofase de 14 horas. Foram utilizadas ninfas de $\boldsymbol{P}$. nigrispinus, no primeiro dia do $2^{\circ}$ estádio, obtidas da criação desse percevejo, que é mantida no laboratório de Entomologia do CCA-UFES. O delineamento experimental adotado foi o inteiramente casualizado, e, com base na alimentação fornecida ao predador, foram utilizados três tratamentos sendo cada um constituído de 60 repetições.

No tratamento um (T1), foram oferecidas lagartas, de quarto estádio, da presa $\boldsymbol{S}$. frugiperda durante toda fase jovem e adulta; no tratamento dois (T2), larvas de $\boldsymbol{T}$. molitor durante toda fase jovem e adulta; e no tratamento três (T3), lagartas de $\boldsymbol{S}$. frugiperda do $2^{\circ}$ ao $4^{\circ}$ instar, e T. molitor no $4^{\circ}$ e $5^{\circ}$ instar e durante toda a fase adulta.

Para cada repetição, uma ninfa de $\boldsymbol{P}$. nigrispinus com sua respectiva presa, foi acondicionada uma placa de Petri com 9,2x1,5cm, contendo na parte superior um chumaço de algodão umedecido com água destilada, para fornecimento de água e umidade e, na parte inferior, papel de filtro cobrindo todo fundo da mesma, com a função de absorver o excesso de umidade. As presas eram substituídas sempre que se encontravam predadas e necrosadas.

Foram feitas observações diárias para se determinar o dia da troca de estádio e, neste dia, esses percevejos eram pesados em balança da marca Chyo, modelo JK 200, para determinar a porcentagem de ganho de peso, que foi feita da seguinte forma: Ganho de peso $=$ Peso final - Peso inicial Logo, se peso inicial $-100 \%$ Então: ganho de peso $\%$ de ganho de peso
Os dados obtidos foram submetidos à analise de variância e as médias comparadas pelo teste de Tukey, a 5\% de probabilidade.

Após o terceiro dia de emergência dos adultos, iniciou-se uma nova etapa de avaliação. Nesta fase, foram formados casais a partir dos adultos obtidos da fase jovem. Estes casais foram acondicionados em potes de $500 \mathrm{ml}$, com $9,5 \mathrm{~cm}$ de altura por $10 \mathrm{~cm}$ de diâmetro, seguindo-se a mesma alimentação, em função dos tratamentos, que estava sendo ministrada ao final da fase inicial. Para fornecimento de água ao inseto e manutenção de umidade, foi colocado um tubo, tipo anestesia, fixado à borda da tampa e tapado em sua extremidade por algodão contendo água destilada. $\mathrm{O}$ recipiente foi envolto no seu interior, por papel do tipo “ofício”, para servir de sítio de oviposição. Foram feitas anotações diárias para avaliar o número de ovos e o dia da morte destes adultos.

Para a porcentagem média de ganho de peso de ninfas de $\boldsymbol{P}$. nigrispinus, não foram observadas diferenças significativas em nenhum dos estádios, e os resultados encontrados mostraram que, no segundo estádio, quando as ninfas foram alimentadas com lagartas de $\boldsymbol{S}$. frugiperda, houve um ganho de 258,93\%, enquanto que, para aquelas que receberam larvas de $\boldsymbol{T}$. molitor, esse foi de $237,54 \%$. No terceiro estádio, larvas de $\boldsymbol{T}$. molitor proporcionaram ganho de 231,45\%, já aquelas alimentadas com $\boldsymbol{S}$. frugiperda, tiveram ganho de $177,68 \%$. No estádio seguinte, o tratamento três (T3), que sofreu mudança de dieta passando a receber larvas de $\boldsymbol{T}$. molitor, apresentou incremento no ganho de peso de $218,81 \%$, e os tratamentos um e dois, apresentaram $200,71 \%$ e $162,92 \%$, respectivamente. No último estádio ninfal, embora SANTOS et al. (1995) e OLIVEIRA et al. (2002) relatem ser o período em que podem ocorrer maiores diferenças entre dietas, devido à formação dos adultos, os resultados encontrados mostram ganho semelhante entre as diferentes dietas utilizadas com 124,68\%, 121,37\%, 110,70\%, para T1, T2, T3 respectivamente (Figura 1).

Nos primeiros dois dias da fase adulta, também não se observou diferença significativa entre os tratamentos, e o ganho de peso foi de 3,35\% (T1), 3,93\% (T2), e de 0,59\%(T3) para o primeiro dia, enquanto que no segundo, esse ganho foi de $8,71 \%$, 4,22\%, 2,78\%, para os tratamentos 1, 2, 3, respectivamente (Figura 1). Segundo OLIVEIRA et al. (2002), o ganho de peso observado nesse período em todos tratamentos está relacionado, com o desenvolvimento do ovário da fêmea.

Com relação ao número de ovos produzidos, percevejos que foram alimentados durante todo o ciclo com lagartas de $\boldsymbol{S}$. frugiperda (T1), tiveram uma maior 


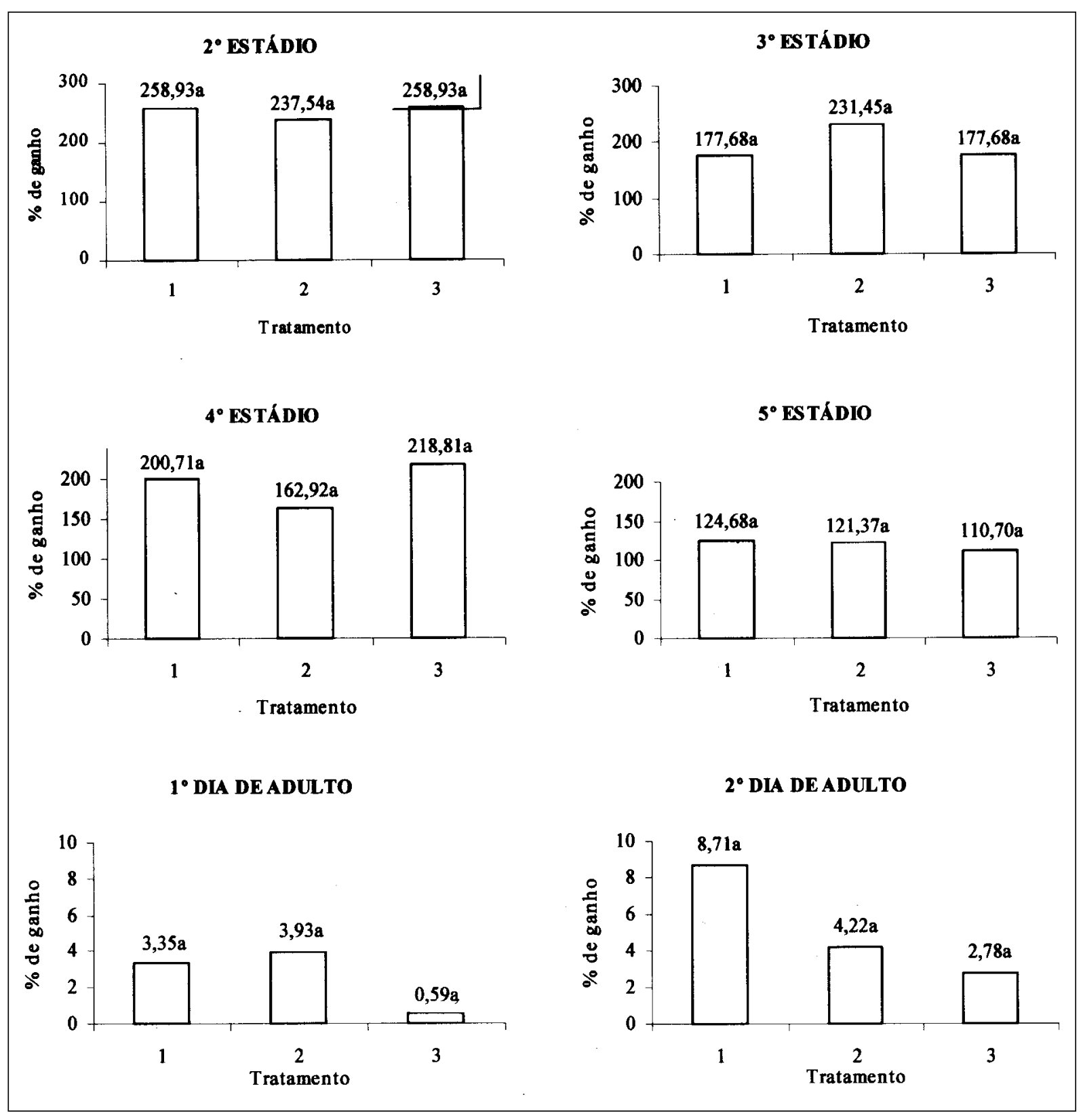

Figura. 1 - Ganho de peso (\%), de fêmeas de Podisus nigrispisnus, no $2^{\circ}$, $3^{\circ}$, $4^{\circ}, 5^{\circ}$ estádios, e adultos no $1^{\circ}$ e $2^{\underline{0}}$ dia de idade, alimentados com: Spodoptera frugiperda (T1); Tenebrio molitor (T2); $S$. frugiperda do $2^{-}$ao $4^{\circ}$ estádios e $T$. molitor do $4^{\circ}$ ao estádio ao $3^{\circ}$ dia de adulto (T3). Médias seguidas de mesma letra minúscula não diferem entre si em nível de $5 \%$ de probabilidade pelo teste Tukey.

produção de ovos num menor período, com $80 \%$ dos ovos até $31^{\circ}$ dia, enquanto que, no tratamento com larvas de $\boldsymbol{T}$. molitor (T2), os mesmos $80 \%$ foram conseguidos somente no $48^{\circ}$ dia, e no tratamento com a combinação de presas (T3) no 45ํ dia (Figura 2).

O ganho de peso de $\boldsymbol{P}$. nigrispinus durante toda a fase ninfal e nos dois primeiros dias da fase adulta, não mostraram diferenças entre os tratamentos, no entanto, observou-se, para os adultos, alimentados com $\boldsymbol{S}$. frugiperda, uma maior produção de ovos num menor período de oviposição. Esse comportamento também foi relatado por OHGUSHI (1996) para Epilachna niponica (Lewis) (Coleoptera: Coccinellidae), e esse autor evidenciou que não existe uma relação entre peso e período reprodutivo. Sendo assim, um fato que poderia explicar esse comportamento de oviposição é a qualidade nutricional do alimento. OLIVEIRA et al. (2002) mostraram que 


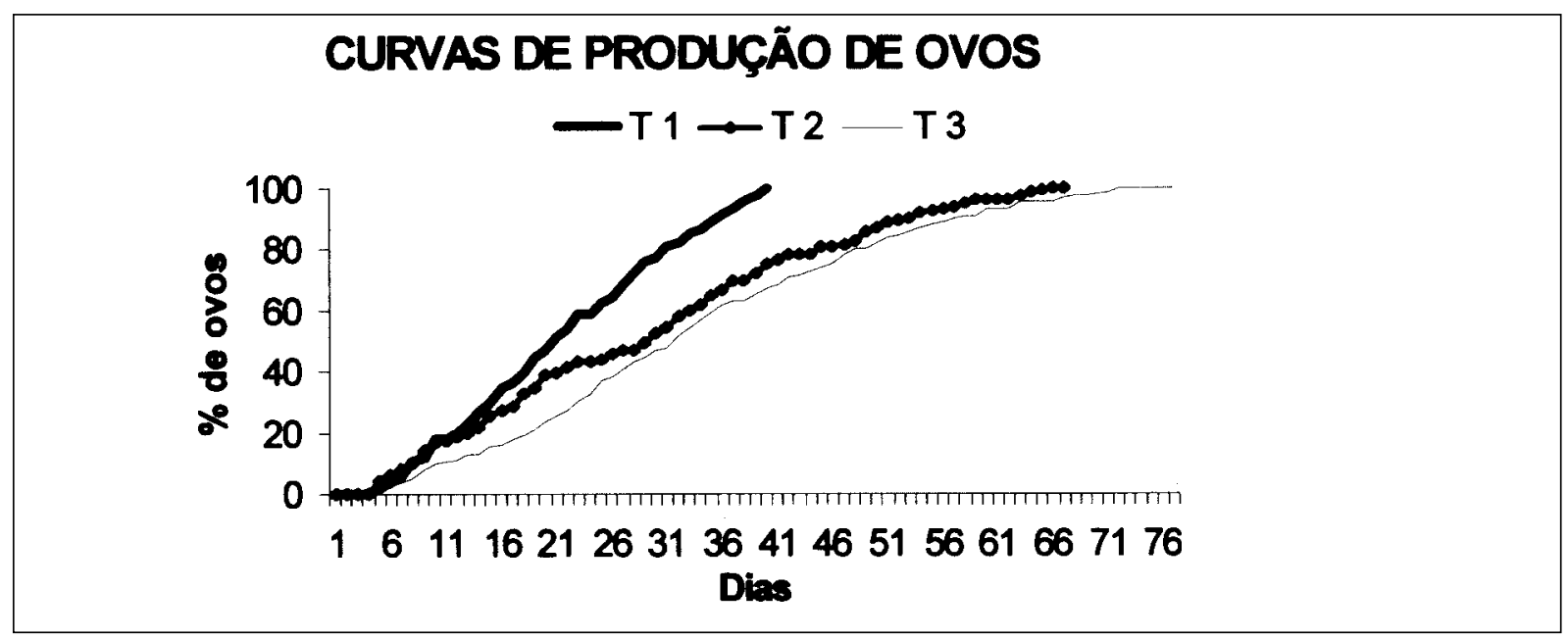

Figura 2 - Curva de porcentagem de ovos, em função do dia de vida (adulta) de fêmeas de $\boldsymbol{P}$. nigrispinus alimentadas com: Spodoptera frugiperda (T1), Tenebrio molitor (T2), durante toda vida; e $S$. frugiperda do $2^{\underline{0}}$ ao $4^{\underline{0}}$ estádios e $\boldsymbol{T}$. molitor do $4^{\underline{0}}$ ao estádio até o fim de sua vida (T3).

fêmeas de $\boldsymbol{P}$. nigrispinus, tiveram uma maior produção de ovos no início da fase adulta quando alimentadas com T. molitor e suplementadas com plantas de algodoeiro, em relação às supridas com tomateiro. Esses autores sugeriram que algodoeiro possui nutrientes que possivelmente complementam as necessidades nutricionais desse predador, sendo assim entendemos que tais necessidades talvez possam ser supridas por lagartas de $\boldsymbol{S}$. frugiperda uma vez que percevejos alimentados com estas apresentaram maior taxa de oviposição num menor período.

Fica evidenciado então que, apesar de não haver diferença no ganho de peso entre os tratamentos, o tipo de presa influenciou o comportamento de oviposição de $\boldsymbol{P}$. nigrispinus e que a utilização da presa S. frugiperda pode viabilizar o aumento da criação massal num menor período.

\section{AGRADECIMENTOS}

Ao CNPq (Conselho Nacional de Desenvolvimento Científico e Tecnológico) pelas bolsas e auxílio concedido e ao professor Dr. José Cola Zanuncio por ceder os percevejos utilizados para iniciar a criação em nosso laboratório.

\section{REFERÊNCIAS BIBLIOGRÁFICAS}

GRAVENA, S.; LARA, F.M. Controle integrado de pragas e receituário agronômico. In: GRAZIANO NETO, F. (eds) Receituário agronômico. São Paulo : Agroedições, 1982. p. 123-161.

HOUSE, H.L. Nutrition of natural enemies. In: RIDGWAY, R.L. \& VISION, S.B. (eds). Biological control by augmentation of natural enemies. New York : Plenum, 1977. p.151-182
OHGUSHI, T. Consequences of adult size for survival and reproductive performance in a herbivorous ladybird beetle. Ecological Entomology, Oxford, v.21, n.1, p.47-551, 1996.

OLIVEIRA, J.E.M. et al. Efeito das plantas do algodoeiro e do tomateiro, como complemento alimentar, no desenvolvimento e na reprodução do predador Podisus nigrispinus (Dallas) (Heteroptera: Pentatomidae). Neotropical Entomology, Londrina, v.31, n.1, p.101-109, 2002.

SANTOS, T.M. et al. Desenvolvimento ninfal de Podisus connexivus Bergroth (Hemiptera: Pentatomidae) alimentado com curuquerê-do-algodoeiro. Pesquisa Agropecuária Brasileira, Brasília, v.30, p.163-167,1995.

THOMAS, D.B. Taxonomic synopsis of the Asopinae Pentatomidae (Heteroptera) of the Western hemisphere. Maryland : Entomology Society of America, 1992. 147p. (Thomas Say Foundation Monography).

TORRES, J.B. et al. Workshop sobre Fitossanidade Florestal do Mer cosul. Santa Maria : CEPEF/FATEC/UFSM, 1996. 81p.

ZANUNCIO, J.C. et al. Fecundidade de fêmeas de Podisus connexivus BERGROTH, 1891 (Hemiptera: Pentatomidae) em diferentes tipos de hospedeiros. Anais da Sociedade Entomologica do Brasil, Londrina, v.20, n.2, p.369-378, 1991.

ZANUNCIO, J.C. et al. Desenvolvimento ninfal de Podisus conexivus Bergroth, 1981 (Hemiptera: Pentatomidae), alimentado com pré-pupas de Psorocampa denticulata (Lepidoptera: Notodontidae) e larvas de Tenebrio molitor (Coleoptera: Tenebrionidae). Revista Brasileira de Entomologia, Curitiba, v.37, n.3, p.523-527, 1993.

ZANUNCIO, J.C. et al. Uma década de estudos com percevejos predadores: Conquistas e desafios. In: Controle Biológico no Brasil: parasitóides e predadores. São Paulo : Manole, 2002. Cap.29, p.495-528. 